\title{
Lidil
}

Revue de linguistique et de didactique des langues

$64 \mid 2021$

Le passif dans la langue parlée

Jean-Marie Fournier, Aimée Lahaussois et Valérie Raby (dir.), Grammaticalia. Hommage à Bernard Colombat

Lyon, ENS Éditions, 2019, 318 p.

Julie Sorba

2 OpenEdition

Journals

Édition électronique

URL : https://journals.openedition.org/lidil/9403

DOI : $10.4000 /$ lidil.9403

ISSN : $1960-6052$

Éditeur

UGA Éditions/Université Grenoble Alpes

Édition imprimée

ISBN : 978-2-37747-315-1

ISSN : $1146-6480$

Référence électronique

Julie Sorba, « Jean-Marie Fournier, Aimée Lahaussois et Valérie Raby (dir.), Grammaticalia. Hommage à

Bernard Colombat », Lidil [En ligne], 64 | 2021, mis en ligne le 01 novembre 2021, consulté le 28

novembre 2021. URL : http://journals.openedition.org/lidil/9403; DOI : https://doi.org/10.4000/lidil.

9403

Ce document a été généré automatiquement le 28 novembre 2021.

(C) Lidil 


\section{Jean-Marie Fournier, Aimée Lahaussois et Valérie Raby (dir.), Grammaticalia. Hommage à Bernard Colombat}

Lyon, ENS Éditions, 2019, 318 p.

Julie Sorba

\section{RÉFÉRENCE}

Jean-Marie Fournier, Aimée Lahaussois et Valérie Raby (dir.), Grammaticalia. Hommage à Bernard Colombat, Lyon, ENS Éditions, 2019, 318 p.

1 Ce volume rend hommage à un « authentique savant » comme l'écrit S. Auroux dans les derniers mots de sa postface. Les vingt-huit contributions qui le constituent, courtes et denses, éclairent chacune une facette du travail du linguistique et de l'historien de la langue latine qu'est le récipiendaire. Les quatorze pages de la rubrique "Travaux et publications de Bernard Colombat» donnent d'ailleurs une idée de la richesse de la production scientifique de ce chercheur qui fut jadis grenoblois. Les éditeurs de l'ouvrage l'ont structuré en quatre parties ou grands postes d'observation de l'histoire de la langue : l'activité de catégorisation se réalisant dans le métadiscours grammatical (première partie « Partie du discours, catégories, notions »); les grammairiens et leurs écrits à différentes époques (deuxième partie « Auteurs et œuvres dans l'histoire de la linguistique »); les processus de transfert d'une tradition descriptive à une autre ou d'une langue à une autre (troisième partie "Transferts ou outillage des langues »); le statut des grammaires, des grammairiens et de leurs pratiques (quatrième partie «Perspectives transversales »).

2 A. Lahaussois interroge, de manière originale, l'utilité de la catégorie des parties du discours pour les linguistes de terrain. En tant que spécialiste d'une langue en danger 
parlée au Népal, elle a fréquenté les manuels de terrain dont l'objectif est d'expliquer " comment collecter des données de première main en travaillant avec des locuteurs " (p. 27). Même si ces ouvrages se présentent plutôt comme des modes d'emploi, ils exercent néanmoins une certaine influence sur leurs utilisateurs en informant plus ou moins la vision qu'ils pourront avoir des unités de la langue dont ils recueillent les données. Au sein de cet ensemble, elle distingue les ouvrages de type «collecte de données " de ceux du type "description et analyse des données». Autant dans les premiers, les termes sont absents ou utilisés comme de simples «étiquettes notionnelles ", autant dans les seconds leur utilisation métalinguistique est pleinement justifiée pour permettre l'analyse.

3 L'étude contrastive franco-italienne proposée par B. Bouard et A. Mosca se focalise sur la signification et la circulation des deux termes attribut et prédicat dans un corpus de grammaires du $\mathrm{xVII}^{\mathrm{e}}$ au $\mathrm{xIX}^{\mathrm{e}}$ siècle. Elles se proposent d'expliquer l'exception française: «[...] en français, on nomme attribut ce qu'ailleurs, dans les langues romanes, mais pas seulement, on nomme prédicat.» (p.76) Il s'agit ainsi de comprendre historiquement comme le linguiste choisit les termes qui lui servent à décrire son objet d'étude, la langue.

Dans sa contribution, C. Pagani-Naudet se donne comme objectif de montrer comment Ramus, à un moment charnière de l'histoire de la langue française, "se pose en champion de l'usage » (p.117). Par sa posture résolument moderne, le savant tente d'instaurer un autre rapport aux données. C'est par le remaniement des exemples qu'il utilise dans sa grammaire de 1572 que la nouveauté se fait discrètement sentir comme l'auteure l'illustre amplement. Cette modernité de l'approche de la langue se rencontre aussi dans la Grammaire générale de Beauzée (1767) dans laquelle J.-M. Fournier et V. Raby examinent précisément la place des langues peu décrites ou peu connues des grammairiens européens de l'époque. C'est ainsi que sont présentés des exemples significatifs du rapport du grammairien à l'archive grammaticale (l'article en suédois, les cas en quechua) qui révèlent que ce sont les données collectées par d'autres et non leurs analyses qui l'intéressent.

Dans «Comment mal traduire une grammaire », K. Ahačič propose une contribution malicieuse qui révèle un cas d'école: la traduction allemande fautive de la partie métalinguistique de la première grammaire de la langue slovène (1758). À la question posée dans le titre, l'une des incohérences les plus fréquentes répond de manière explicite : «[...] quand la règle ou l'exemple allemand et la règle ou l'exemple slovène ne correspondent ni l'un ni l'autre à l'état des choses en allemand et en slovène " (p. 176). Les nombreux exemples cités permettent de constater que les traductions à répétition du latin au slovène puis à l'allemand brouillent finalement la clarté du propos initial.

6 M. Baratin propose une réflexion sur « La traduction selon Justinien, ou la littéralité en milieu bilingue » pour comprendre ce que signifie le terme kata poda qui qualifie la seule traduction autorisée du texte juridique latin du Digeste, recueil de lois promulguées en 533. Il s'agit en fait d'une traduction tellement littérale qu'elle reproduit dans la langue d'arrivée (le grec) la syntaxe de la langue de départ (le latin). L'étrangeté de ce procédé conduit, somme toute, à rendre inintelligible la traduction. Néanmoins, celui-ci est rendu possible par le contexte bilingue dans lequel «la présence [de la langue de départ] demeure dans le caractère non naturel de la formulation dans la langue d'arrivée » (p. 255). 
7 Le lectorat académique pourra trouver dans la lecture de ce volume de nombreuses autres occasions de faire écho aux travaux de Bernard Colombat que celles que nous avons sélectionnées ici. Chacune d'entre elles apporte une pierre d'érudition à l'édifice, patiemment érigé, de l'histoire de la langue et des langues.

\section{AUTEURS}

\section{JULIE SORBA}

Univ. Grenoble Alpes, LIDILEM, 38000 Grenoble, France 